\title{
On the asymptotic uniqueness of bargaining equilibria
}

\author{
Citation for published version (APA):
}

Predtetchinski, A. (2009). On the asymptotic uniqueness of bargaining equilibria. METEOR, Maastricht University School of Business and Economics. METEOR Research Memorandum No. 021 https://doi.org/10.26481/umamet.2009021

Document status and date:

Published: 01/01/2009

DOI:

10.26481/umamet.2009021

Document Version:

Publisher's PDF, also known as Version of record

\section{Please check the document version of this publication:}

- A submitted manuscript is the version of the article upon submission and before peer-review. There can be important differences between the submitted version and the official published version of record.

People interested in the research are advised to contact the author for the final version of the publication, or visit the DOI to the publisher's website.

- The final author version and the galley proof are versions of the publication after peer review.

- The final published version features the final layout of the paper including the volume, issue and page numbers.

Link to publication

\footnotetext{
General rights rights.

- You may freely distribute the URL identifying the publication in the public portal. please follow below link for the End User Agreement:

www.umlib.nl/taverne-license

Take down policy

If you believe that this document breaches copyright please contact us at:

repository@maastrichtuniversity.nl

providing details and we will investigate your claim.
}

Copyright and moral rights for the publications made accessible in the public portal are retained by the authors and/or other copyright owners and it is a condition of accessing publications that users recognise and abide by the legal requirements associated with these

- Users may download and print one copy of any publication from the public portal for the purpose of private study or research.

- You may not further distribute the material or use it for any profit-making activity or commercial gain

If the publication is distributed under the terms of Article $25 \mathrm{fa}$ of the Dutch Copyright Act, indicated by the "Taverne" license above, 


\section{Maastricht University}

Arkadi Predtetchinski

On the asymptotic uniqueness of bargaining equilibria

RM/09/021

\section{METEOR}

Faculty of Economics and Business Administration Maastricht Research School of Economics

of Technology and Organization

\section{P.O. Box 616}

NL - 6200 MD Maastricht

The Netherlands 


\title{
On the asymptotic uniqueness of bargaining equilibria
}

\author{
Arkadi Predtetchinski*
}

May 11, 2009

\begin{abstract}
The paper studies the model of multilateral bargaining over the alternatives represented by points in the $m$-dimensional Euclidean space. Proposers are chosen randomly and the acceptance of a proposal requires the unanimous approval of it by all the players. The focus of the paper is on the asymptotic behavior of subgame perfect equilibria in pure stationary strategies (called bargaining equilibria) as the breakdown probability tends to zero. Bargaining equilibria are said to be asymptotically unique if the limit of a sequence of bargaining equilibria as the breakdown probability tends to zero is independent of the choice of the sequence and is uniquely determined by the primitives of the model. We show that the limit of any sequence of bargaining equilibria is a zero point of the so-called linearization correspondence. The asymptotic uniqueness of bargaining equilibria is then deduced in each of the following cases: (1) $m=n-1$, where $n$ is the number of players, (2) $m=1$, and (3) in the case where the utility functions are quadratic, for each $1 \leq m \leq n-1$. In each case the linearization correspondence is shown to have a unique zero. Result 1 has been established earlier in Miyakawa [13] and Laruelle and Valenciano [3]. Result 2 is subsumed by the result in Predtetchinski [14]. Result 3 is new.
\end{abstract}

JEL classification codes: C78.

Keywords: Bargaining, subgame perfect equilibrium, stationary strategies.

${ }^{*}$ Department of Economics, Maastricht University, P.O. Box 616, 6200 MD Maastricht, The Netherlands. E-mail: A.Predtetchinski@algec.unimaas.nl. The author is most thankful to P. Jean-Jacques Herings for his many comments on the subject. The author acknowledges the financial support of the Dutch Science Foundation. 


\section{The model}

This paper studies strategic bargaining as in the model of Banks and Duggan [1]. The alternatives are represented by the points in the Euclidean space. At the beginning of each period of time a random draw determines whether bargaining continues or the game terminates. If the game terminates, the players receive their disagreement payoffs. If bargaining continues, a player is randomly chosen to make a proposal. The chosen player proposes one alternative, and the players vote on the proposal. Each player can either reject or accept the proposal. Voting proceeds sequentially, with the sequence of responses being fixed throughout the game. If the proposal is unanimously accepted it is implemented and the game ends. Otherwise, the next period begins. The recognition and the breakdown probabilities are exogenously given.

The focus of the paper is on the asymptotic uniqueness of subgame perfect equilibria in pure stationary strategies which we call bargaining equilibria. Consider a sequence of bargaining equilibria as the breakdown probability tends to zero. If the equilibrium proposal of some player $i$ converges to the alternative $x$, so does the equilibrium proposal of any other player $j$. If the point $x$ is uniquely determined by the primitives and is independent of the choice of the sequence of bargaining equilibria, we say that the equilibria are asymptotically unique. Asymptotic uniqueness of equilibria is a very attractive property of a bargaining model. Even though it does not guarantee the uniqueness of equilibria for any given breakdown probability, it does imply that the multiplicity of equilibria becomes insignificant as the breakdown probability becomes small.

The asymptotic uniqueness of equilibria has been so far shown (a) for the onedimensional model of bargaining and (b) for the games where the set of efficient utility payoffs is a $(n-1)$-dimensional set, where $n$ denotes the number of players. We discuss the respective contributions in turn.

The one-dimensional model of bargaining is a game where the space of alternatives is a subset of the real line. Sufficient condition for the (asymptotic) uniqueness of bargaining equilibria in such models have been given in Cho and Duggan [5], Cardona and Ponsatí [4], Herings and Predtetchinski [8] and in Predtetchinski [14]. The asymptotic uniqueness of equilibria in the one-dimensional model has been shown under very mild assumptions on the primitives. In particular, Cardona and Ponsatí [4] establish the asymptotic uniqueness in a version of the model where the proposer is chosen according to some deterministically rotating scheme, while Predtetchinski [14] studies a game with time-invariant recognition probabilities (as in this paper). In either case, all that is required of the utility functions is concavity and single-peakedness. More restrictive assumptions have to be invoked to obtain the uniqueness of equilibria in a fixed game (see $[4,5,8]$ ). The examples of onedimensional games of bargaining with multiple equilibria are given in Cho and Duggan [5] and in Kalandrakis [9].

We now turn to the second group of contributions: Miyakawa [13], Laruelle and Valenciano [3], Eraslan [6], Kultti and Vartiainen [10]. These are works where the set of efficient payoff vectors is assumed to be of dimension $n-1$, where $n$ is the number of players. Since $n-1$ is the highest dimension the efficient payoff vectors can have, we call such games 
full-dimensional. An important example of a full-dimensional game is the divide-thedollar game where the player's utility is assumed to depend only on the amount of money allocated to that player.

Miyakawa [13] and Laruelle and Valenciano [3] show that under the assumption of full-dimensionality as the breakdown probability tends to zero the bargaining equilibria converge to the asymmetric Nash bargaining solution. This is the point that maximizes the geometric weighted mean of the payoffs with the weights equal to the respective recognition probabilities. These findings generalize an earlier result of Binmore et al [2] for the two-player case. A related result is given in Kultti and Vartiainen [10] who obtain the asymmetric Nash bargaining solution as a limit of the Von Neumann-Morgenstern stable set as the discount factor vanishes. The stable set is defined with respect to a dominance relation where an alternative $x$ dominates an alternative $y$ if some player prefers $x$ over $y$ even with a one-period delay. Eraslan [6] establishes the uniqueness of equilibria in the divide-the-dollar game under the assumption that the utility functions are linear.

Also related is the work of Kalandrakis [9] where local uniqueness of stationary equilibria has been shown for the model in Banks and Duggan [1] in its full generality. Merlo and Wilson [11, 12], and Eraslan and Merlo [7] study a more general model of bargaining where the set of feasible agreements changes stochastically over time.

The contribution of this paper is twofold. We provide a unified approach to the problem of asymptotic uniqueness of bargaining equilibria in the model of Banks and Duggan [1] and deduce the asymptotic uniqueness in the one-dimensional and in the full-dimensional cases from our general result. We also identify a class of games where bargaining equilibria are asymptotically unique irrespectively of the dimension of the set of alternatives or efficient payoff vectors.

In more detail our results are as follows. We show that the limit of any sequence of bargaining equilibria is a zero point of the so-called linearization correspondence. Asymptotic uniqueness of equilibria is then established in each of the following cases: (1) $m=n-1$, where $m$ is the dimensionality of the set of alternatives, (2) $m=1$, and (3) the case where the utility functions are quadratic, for each $1 \leq m \leq n-1$. In each case the asymptotic uniqueness of equilibria is proven by showing that the linearization correspondence has a unique zero.

Result 1 for the full-dimensional case is the same as in Miyakawa [13] and Laruelle and Valenciano [3] with the asymmetric Nash bargaining solution being the unique limit of bargaining equilibria. Result 2 on the one-dimensional model is somewhat weaker than in Predtetchinski [14] due to the fact that we restrict our attention to the unanimity acceptance rule. It is complementary to the asymptotic uniqueness result in Cardona and Ponsatí [4] who consider a game where the proposer is determined by a fixed rotation rule. Result 3 for the quadratic utility functions is new. To the best of my knowledge, it is the only result on the asymptotic uniqueness of bargaining equilibria outside the extreme cases $m=1$ and $m=n-1$.

The rest of the paper is organized as follows. In Section 2 the game of bargaining is presented and the assumptions on the primitives are discussed. In Section 3 it is shown that the difference between the equilibrium proposals of any two players tends to zero 
as the breakdown probability becomes negligible. In Section 4 the equilibrium conditions are linearized to obtain the so-called linearization correspondence. It is shown that the limit of any sequence of bargaining equilibria is a zero of the linearization correspondence. Sections 5, 6 and 7 establish the uniqueness of the limit of bargaining equilibria in the one-dimensional case, the full-dimensional case, and the case with quadratic utilities, respectively.

\section{The model}

Consider a situation where the players have to agree on a choice of a point from the $m_{-}^{-}$ dimensional Euclidean space $\mathbb{R}^{m}$. The points of $\mathbb{R}^{m}$ will be referred to as alternatives. The set of players will be denoted by $N$, with $n$ being the number of players. The utility of an alternative $x$ to player $i$ is $u_{i}(x)$. There is a probability distribution $\mu$ on the set $N$. The probability $\mu_{i}$ is the probability for player $i$ to be chosen as a proposer and is called the recognition probability of player $i$. The model is specified by the choice of $m$, the dimension of the set of alternatives, the set of players $N$, the recognition probabilities $\mu$ and the utility functions $u_{i}$ for $i$ in $N$.

We now discuss our assumptions on the utility functions.

(A1) The function $u_{i}$ is concave and continuously differentiable.

(A2) The set $X=\left\{x \in \mathbb{R}^{m} \mid u_{i}(x) \geq 0\right\}$ is compact and it contains the alternative $x$ such that $u_{i}(x)>0$ for each $i \in N$.

Now the alternative $x \in \mathbb{R}^{m}$ is said to be weakly Pareto efficient if there is no other alternative $\bar{x}$ such that $u_{i}(\bar{x})>u_{i}(x)$ for each $i \in N$. The alternative $x$ is said to be Pareto efficient if there is no other point $\bar{x}$ such that $u_{i}(\bar{x}) \geq u_{i}(x)$ for each $i \in N$ and $u_{k}(\bar{x})>u_{k}(x)$ for some $k \in N$. We write $d u_{i}(x)$ to denote the vector of partial derivatives of $u_{i}$ at the point $x$. The inner product of the vectors $z$ and $x$ is denoted by $\langle x, z\rangle=x^{1} z^{1}+\cdots+x^{m} z^{m}$.

(A3) Each weakly Pareto-efficient alternative is Pareto efficient.

(A4) If $x$ is a Pareto-efficient alternative and $z$ a vector such that $\left\langle d u_{i}(x), z\right\rangle=0$ for each $i \in N$, then $z=0$.

Assumption (A3) is satisfied if all utility functions are strictly concave. Assumption (A4) guarantees that there is a one-to-one relationship between the efficient alternatives and payoffs: no two distinct Pareto-efficient alternatives give the same payoff to every player. In this sense, no alternative is "redundant".

Given a continuation probability $\delta \in[0,1)$ we define the game of bargaining $\Gamma(\delta)$ as follows. The game starts in period zero. At the beginning of each period a random draw determines whether bargaining continues of the game terminates, in which case each player receives the disagreement payoff of zero. The probability that bargaining continues is $\delta$. If bargaining does continue, a player is randomly drawn from the set $N$ to make a 
proposal. Player $i$ is chosen with probability $\mu_{i}$. The chosen player proposes an alternative $x$ from $\mathbb{R}^{m}$. All players (including the proposer) respond sequentially, with the sequence of responses being fixed throughout the game. Each responder can either accept or reject the current proposal. If all players accept the proposal, the game terminates and the proposal $x$ is implemented, with player $i$ receiving the payoff of $u_{i}(x)$. Otherwise, the next period begins.

The game tree of $\Gamma(\delta)$ is similar to that in Banks and Duggan [1] with the difference that in $\Gamma(\delta)$ the players vote sequentially, while in [1] the votes are cast simultaneously. We insist that players vote sequentially to rule out coordination problems in the response stage of the game, for example a situation when any alternative is unanimously rejected independently of preferences. Furthermore, we focus on the unanimity acceptance rule to make sure that subgame perfect equilibria in pure stationary exist (see Theorem 2 in Banks and Duggan [1]).

We shall restrict our attention to pure stationary strategies by which we mean the strategies such that (a) a proposal of any player is independent of the history of play and (b) the reaction of a player to a proposal only depends on the proposal itself. Thus a stationary strategy of player $i$ consists of a proposal $x_{i}$ and an acceptance set $A_{i}$. Player $i$ proposes the point $x_{i}$ whenever player $i$ is chosen to be a proposer and he accepts a proposal $x$ if and only if $x$ is an element of the set $A_{i}$. A joint stationary strategy $\sigma=\left(x_{\bullet}, A_{\bullet}\right)$ induces the social acceptance set $A=\cap A_{i}$. A proposal $x$ is implemented if and only if it is an element of the social acceptance set $A$.

Consider the case where the proposal $x_{i}$ of each player $i$ lies in the social acceptance set $A$. In this case each proposal is accepted without delay. The expected utility $y_{i}$ of player $i$ can then be computed as a weighted sum of $u_{i}\left(x_{k}\right)$, where the weights equal to the respective recognition probabilities. Player $i$ 's continuation payoff when a proposal is turned down is given by $\delta y_{i}$. Therefore, player $i$ accepts a proposal $x$ if and only if $u_{i}(x) \geq \delta y_{i}$. Since all proposals in the set $A$ pass, player $i$ proposes a point $x_{i}$ that maximizes $i$ 's utility on $A$. These considerations motivate the following definition.

Definition 1 A joint stationary strategy $\sigma=\left(x_{\bullet}, A_{\bullet}\right)$ is a bargaining equilibrium of the game $\Gamma(\delta)$ if

$$
\begin{gathered}
x_{i}=\arg \max _{x \in A} u_{i}(x) \text { for each } i \in N, \\
y_{i}=\sum_{k \in N} \mu_{k} u_{i}\left(x_{k}\right) \text { for each } i \in N, \text { where } \\
A_{i}=\left\{x \in X \mid u_{i}(x) \geq \delta y_{i}\right\} \text { for each } i \in N, \\
A=\cap A_{i} .
\end{gathered}
$$

One can show that a bargaining equilibrium thus defined is a subgame perfect equilibrium of the game $\Gamma(\delta)$. Conversely, given a subgame perfect equilibrium of the game $\Gamma(\delta)$ in pure stationary strategies there exists a bargaining equilibrium with the same equilibrium proposals and the same expected payoffs. Under the maintained assumptions the existence of bargaining equilibrium follows from Theorem 2 in Banks and Duggan [1]. 


\section{The limit of bargaining equilibria}

In this section we establish that as the continuation probability $\delta$ tends to one, the distance between the equilibrium proposals of any two players converges to zero. Thus if the proposal of player $i$ converges to the point $x$ as $\delta$ tends to one, so does the proposal of any other player $j$. Any such point $x$ is called the limit of a sequence of bargaining equilibria.

Let

$$
S=\left\{i \in N \mid \mu_{i}>0\right\} \text { and } \nu=\min _{i \in S}\left\{\mu_{i}\right\} .
$$

Proposition 3.1, 3.2, and 3.3 are the auxiliary results we need for the proof of Theorem 1.

Proposition 3.1 Let $\left(x_{\bullet}, A_{\bullet}\right)$ be a bargaining equilibrium of the game $\Gamma(\delta)$ inducing the expected payoffs $y_{\bullet}$ and the social acceptance set $A$. Then $A \subset X$.

Proof. Each point $x_{i}$ is an element of the set $A$, so $u_{i}\left(x_{k}\right) \geq \delta y_{i}$ for each $k \in N$. Furthermore, $y_{i}=\sum_{k \in N} \mu_{k} u_{i}\left(x_{k}\right)$. It follows that $y_{i} \geq \delta y_{i}$ or equivalently, $(1-\delta) y_{i} \geq 0$. Since $\delta \in[0,1)$, we have $y_{i} \geq 0$. The result follows since for a point $x$ of $A$ we have $u_{i}(x) \geq \delta y_{i} \geq 0$.

Since $X$ is assumed to be a compact set by (A2), there exists a real number $c$ such that $u_{i}(x) \leq c$ for each $x \in X$.

Proposition 3.2 Let $\left(x_{\bullet}, A_{\bullet}\right)$ be a bargaining equilibrium of the game $\Gamma(\delta)$ inducing the expected payoffs $y_{\bullet}$ and the social acceptance set $A$. Then $\left|u_{k}\left(x_{i}\right)-u_{k}\left(x_{j}\right)\right| \leq(1-\delta) c / \nu$ for each $k \in N$ and each $(i, j) \in S \times S$.

Proof. Fix a player $k \in N$. The proposal $x_{i}$ of any player $i$ is in the social acceptance set $A$ which is contained in the set $X$ by Proposition 3.1. Therefore, $u_{k}\left(x_{i}\right) \leq c$ for each $i \in N$ and $y_{k} \leq c$ for each $k \in N$. Furthermore, $u_{k}\left(x_{i}\right) \geq \delta y_{k}$. For each $i \in S$ there is a chain of inequalities:

$$
\begin{aligned}
y_{k} & =\sum_{l \in S} \mu_{l} u_{k}\left(x_{l}\right) \\
& \geq \mu_{i} u_{k}\left(x_{i}\right)+\left(1-\mu_{i}\right) \delta y_{k} \\
& =\delta y_{k}+\mu_{i}\left(u_{k}\left(x_{i}\right)-\delta y_{k}\right) \\
& \geq \delta y_{k}+\nu\left(u_{k}\left(x_{i}\right)-\delta y_{k}\right) \\
& =\nu u_{k}\left(x_{i}\right)+(1-\nu) \delta y_{k} .
\end{aligned}
$$

We conclude that

$$
\delta y_{k} \leq u_{k}\left(x_{i}\right) \leq(1-\delta+\nu \delta) y_{k} / \nu \text { for each } i \in S .
$$

Therefore, for each $(i, j) \in S \times S$ we have

$$
\left|u_{k}\left(x_{i}\right)-u_{k}\left(x_{j}\right)\right| \leq(1-\delta+\nu \delta) y_{k} / \nu-\delta y_{k}=(1-\delta) y_{k} / \nu \leq(1-\delta) c / \nu .
$$


We now show that there is a one-to-one relation between the Pareto-efficient alternatives and the payoffs: no pair of distinct Pareto-efficient alternatives give the same payoff to every player.

Proposition 3.3 Let $x$ and $\bar{x}$ be Pareto-efficient alternatives. If $u_{i}(x)=u_{i}(\bar{x})$ for each $i \in N$, then $x=\bar{x}$.

Proof. Let $x^{t}=(1-t) x+t \bar{x}$ for $t \in[0,1]$. By the concavity of the utility function $u_{i}\left(x^{t}\right) \geq u_{i}(x)$ for each $i \in N$. Since $x$ is a Pareto-efficient alternative, we must have $u_{i}\left(x^{t}\right)=u_{i}(x)$ for each $i \in N$. But then $0=\lim _{t \downarrow 0}\left[u_{i}\left(x^{t}\right)-u_{i}(x)\right] / t=\left\langle d u_{i}(x), \bar{x}-x\right\rangle$ for each $i \in N$. By Assumption (A4), we must have $\bar{x}-x=0$.

We are now in a position to state the main result of the section.

Theorem 1 For each natural $r$ let $\left(x_{\bullet}^{r}, A_{\bullet}^{r}\right)$ be a bargaining equilibrium of the game $\Gamma\left(\delta^{r}\right)$ inducing the continuation payoffs $y_{\bullet}^{r}$ and the social acceptance set $A^{r}$. If $\delta^{r} \longrightarrow 1$ then $\left\|x^{r}-\bar{x}^{r}\right\| \longrightarrow 0$ for each sequence $\left(x^{r}, \bar{x}^{r}\right) \in A^{r} \times A^{r}$. Furthermore, if $x^{r} \longrightarrow x$, then $x$ is a Pareto-efficient alternative.

Proof. By Proposition 3.1 the sequence $\left\{x_{i}^{r}\right\}$ is entirely contained in the set $X$ which is compact by Assumption (A2). We can therefore assume without loss of generality that for every $i \in S$ the sequence $\left\{x_{i}^{r}\right\}$ is converging, say to a point $x_{i}$. We first show that the points $x_{i}$ for $i \in S$ are the same.

By the continuity of the utility functions $u_{k}\left(x_{i}^{r}\right) \longrightarrow u_{k}\left(x_{i}\right)$. By Proposition 3.2 $\left|u_{k}\left(x_{i}^{r}\right)-u_{k}\left(x_{j}^{r}\right)\right|<\left(1-\delta^{r}\right) c / \nu$ for each pair $(i, j) \in S \times S$ and each $k \in N$. It follows that $u_{k}\left(x_{i}\right)=u_{k}\left(x_{j}\right)$ for each $(i, j) \in S \times S$ and each $k \in N$. It follows that $y_{k}^{r}=\sum_{l \in S} \mu_{l} u_{k}\left(x_{l}^{r}\right) \longrightarrow u_{k}\left(x_{i}\right)$ for each $i \in S$ and $k \in N$.

The alternative $x_{i}$ for each $i \in S$ is weakly Pareto-efficient. Suppose not. Then there exists an alternative $\dot{x}$ such that $u_{k}(\dot{x})>u_{k}\left(x_{i}\right)$ for each $k \in N$. But then for $n$ large enough $u_{k}(\dot{x})>u_{k}\left(x_{i}^{r}\right)$ for each $k \in N$. Since $u_{k}\left(x_{i}^{r}\right) \geq \delta^{r} y_{k}^{r}$, the point $\dot{x}$ is in the set $A^{r}$, which contradicts the fact that the equilibrium proposal $x_{i}^{r}$ of player $i$ maximizes player $i$ 's utility on $A^{r}$. Thus $x_{i}$ is weakly Pareto-efficient for each $i \in S$. By Assumption (A3) it is also Pareto-efficient. Thus the alternatives $x_{i}$ are Pareto-efficient and give the same utility to each player. It follows from Proposition 3.3 that the point $x_{i}$ is the same for each $i \in S$. Let $x_{i}=\bar{x}$ for each $i \in S$.

We now show that $x^{r} \longrightarrow \bar{x}$ for each sequence $x^{r} \in A^{r}$. Without loss of generality assume that $x^{r} \longrightarrow x$. Since $u_{k}\left(x^{r}\right) \geq \delta^{r} y_{k}^{r}$ for each $n$, in the limit $u_{k}(x) \geq u_{k}(\bar{x})$ for each $k \in N$. Since the alterative $\bar{x}$ has been shown to be Pareto-efficient, we must have $u_{k}(x)=u_{k}(\bar{x})$ for each $k \in N$. It follows from Proposition 3.3 that $x=\bar{x}$, as desired.

Definition 2 A point $x$ as in Theorem 1 is called the limit of a sequence of bargaining equilibria. Bargaining equilibria are said to be asymptotically unique if the limit of a sequence of bargaining equilibria is unique. 


\section{The linearization correspondence}

Consider a limit $x$ of a sequence of bargaining equilibria. The key insight into the nature of the point $x$ comes from the analysis of the limits of the sequence $\left[x_{i}-E\left(x_{\bullet}\right)\right] /(1-\delta)$, where $E\left(x_{\bullet}\right)$ denotes the expected equilibrium proposal. We are able to characterize these limits as a function of $x$. Loosely speaking, this exercise amounts to linearizing the conditions of Definition 1 around the point $\delta=1$. The condition that the sum of these limits weighted with the recognition probabilities be equal to zero translates into the condition that the point $x$ be a zero of the linearization correspondence.

We already know that as the breakdown probability tends to zero, the distance between the equilibrium proposals of any two players converges to zero. Proposition 4.1 below adds to this result that the speed of convergence is at least $1-\delta$. In other words, the difference of $x_{i}$ and $x_{j}$ tends to zero rapidly enough for the ratio $\left(x_{i}-x_{j}\right) /(1-\delta)$ to remain bounded.

Proposition 4.1 For each $n$ let $\left(x_{\bullet}^{r}, A_{\bullet}^{r}\right)$ be a bargaining equilibrium of the game $\Gamma\left(\delta^{r}\right)$ inducing the continuation payoffs $y_{\bullet}^{r}$ and the social acceptance set $A^{r}$. Suppose that $\delta^{r} \longrightarrow 1$ and $x^{r} \longrightarrow x$ for $x^{r} \in A^{r}$. Let

$$
d_{i j}^{r}=\left[x_{i}^{r}-x_{j}^{r}\right] /\left(1-\delta^{r}\right) .
$$

Then for each $(i, j) \in S \times S$ the sequence $\left\{d_{i j}^{r}\right\}$ is bounded.

Proof. Suppose not. Replacing, if necessary, the sequence by a subsequence, we can assume that $\left\|d^{r}\right\|$ converges to infinity. (As $i$ and $j$ are fixed we omit the subscript $i j$ ). By the concavity of the function $u_{k}$ there are the inequalities

$$
\left\langle d u_{k}\left(x_{i}^{r}\right), x_{i}^{r}-x_{j}^{r}\right\rangle \leq u_{k}\left(x_{i}^{r}\right)-u_{k}\left(x_{j}^{r}\right) \leq\left\langle d u_{k}\left(x_{j}^{r}\right), x_{i}^{r}-x_{j}^{r}\right\rangle .
$$

Dividing by $\left(1-\delta^{r}\right)\left\|d^{r}\right\|$ yields the inequalities

$$
\frac{\left\langle d u_{k}\left(x_{i}^{r}\right), d^{r}\right\rangle}{\left\|d^{r}\right\|} \leq \frac{1}{\left\|d^{r}\right\|} \frac{\left[u_{k}\left(x_{i}^{r}\right)-u_{k}\left(x_{j}^{r}\right)\right]}{1-\delta^{r}} \leq \frac{\left\langle d u_{k}\left(x_{j}^{r}\right), d^{r}\right\rangle}{\left\|d^{r}\right\|} .
$$

By Proposition $3.2\left|u_{k}\left(x_{i}^{r}\right)-u_{k}\left(x_{j}^{r}\right)\right| /\left(1-\delta^{r}\right) \leq c / \nu$ for each $k$. By the supposition $1 /\left\|d^{r}\right\| \longrightarrow 0$. By the continuity of the map $d u_{i}$ we have $d u_{k}\left(x_{i}^{r}\right) \longrightarrow d u_{k}(x)$ and $d u_{k}\left(x_{j}^{r}\right) \longrightarrow d u_{k}(x)$. The sequence $d^{r} /\left\|d^{r}\right\|$ lies in the unit sphere, and therefore has a convergent subsequence. Again replacing the sequence by a subsequence we can assume that $d^{r} /\left\|d^{r}\right\|$ converges to a non-zero vector $z$. Then taking the limit in the above inequality as $n$ goes to infinity, we obtain $\left\langle d u_{k}(x), z\right\rangle=0$. Since $k$ is an arbitrary element of $N$, we arrive at a contradiction with Assumption (A4).

Notice that Proposition 4.1 refers only to the players with positive probabilities of recognition. Indeed, the sequence $\left(x_{i}^{r}-x_{j}^{r}\right) /\left(1-\delta^{r}\right)$ need not be bounded if either players $i$ or $j$ have zero recognition probability. We illustrate this point by an example. 
Let $X=[0,1]$ and $N=\{0,1\}$. The utility functions are $u_{0}(x)=1-x$ and $u_{1}(x)=$ $1-(1-x)^{2}$. Thus 0 is player 0 's ideal point while 1 is the ideal point of player 1 . Suppose now the recognition probability of player 0 is 0 . It is easy to see that the interval $A=[1-\sqrt{1-\delta}, 1]$ is the social acceptance set in a bargaining equilibrium of $\Gamma(\delta)$. In this equilibrium player 0 proposes the left endpoint of $A$ and player 1 proposes the right endpoint. The expected utility for player 0 is 0 and for player 1 is 1 . We see that $\left(x_{1}-\right.$ $\left.x_{0}\right) /(1-\delta)=1 / \sqrt{1-\delta}$ goes to infinity as $\delta$ goes to one.

Proposition 4.2 Consider the functions $h_{i}^{r}, h_{i}: \mathbb{R}^{m} \rightarrow \mathbb{R}$ for each $i \in N$ and each natural $r$. Define the sets $C^{r}=\cap\left\{x \in \mathbb{R}^{m} \mid h_{i}^{r}(x) \geq 0\right\}$ and $C=\cap\left\{x \in \mathbb{R}^{m} \mid h_{i}(x) \geq 0\right\}$, where the intersection is taken over all $i$ in $N$. Suppose that $h_{i}^{r}\left(x^{r}\right) \longrightarrow h_{i}(x)$ whenever $x^{r} \longrightarrow x$. Suppose also that the function $h_{i}$ is concave and that there exists an $\bar{x}$ such that $h_{i}(\bar{x})>0$ for each $i$. Then (a) if $x^{r} \in C^{r}$ and $x^{r} \longrightarrow x$, then $x \in C$ and conversely (b) given a point $x \in C$ there exists a sequence $x^{r} \in C^{r}$ such that $x^{r} \longrightarrow x$.

Proof. Part (a) is obvious. We prove (b). Consider first any point $x$ such that $h_{i}(x)>0$ for each $i \in N$. Then $x \in C^{r}$ for $r$ large enough. For if not, there would exist an $i$ such that $h_{i}^{r_{k}}(x)<0$ for all $k$ implying that $h_{i}(x) \leq 0$, a contradiction.

Now let $x$ be an arbitrary point of $C$. The sequence of points $x^{q}=(\bar{x}+(q-1) x) / q$ converges to the point $x$ and $h\left(x^{q}\right)>0$ for each $q$. By the previous paragraph, each point $x^{q}$ is contained in the set $C^{r}$ for $r$ large. That is, for each $q$ there exists a number $m(q)$ such that $x^{q} \in C^{r}$ whenever $r \geq m(q)$. Without loss of generality assume that $m$ is increasing and define the sequence $\left\{\bar{x}^{r}\right\}$ by letting $\bar{x}^{r}=x^{q}$ if $m(q) \leq r<m(q+1)$. Then $x^{r} \in C^{r}$ for each $r$ and $x^{r} \longrightarrow x$.

For each $x \in X$ define

$$
Z(x)=\bigcap_{i \in N}\left\{z \in \mathbb{R}^{m} \mid\left\langle d u_{i}(x), z\right\rangle+u_{i}(x) \geq 0\right\} .
$$

Proposition 4.3 For each $r$ let $\left(x_{\bullet}^{r}, A_{\bullet}^{r}\right)$ be a bargaining equilibrium of the game $\Gamma\left(\delta^{r}\right)$ inducing the continuation payoffs $y_{\bullet}^{r}$ and the social acceptance set $A^{r}$. Suppose that $\delta^{r} \longrightarrow 1$ and $x^{r} \longrightarrow x$ for $x^{r} \in A^{r}$. Let

$$
Z^{r}=\left[A^{r}-E\left(x_{\bullet}^{r}\right)\right] /\left(1-\delta^{r}\right) \text {, where } E\left(x_{\bullet}^{r}\right)=\sum_{i \in S} \mu_{i} x_{i}^{r}
$$

Then (a) if $z^{r} \in Z^{r}$ and $z^{r} \longrightarrow z$, then $z \in Z(x)$ and conversely (b) given a point $z \in Z(x)$ there exists a sequence $z^{r} \in Z^{r}$ such that $z^{r} \longrightarrow z$.

Proof. We apply Proposition 4.2. Notice that $Z^{r}=\cap\left\{z \in \mathbb{R}^{m} \mid h_{i}^{r}(z) \geq 0\right\}$ and $Z=\cap\left\{z \in \mathbb{R}^{m} \mid h_{i}(z) \geq 0\right\}$, where the functions $h_{i}^{r}, h_{i}: \mathbb{R}^{m} \longrightarrow \mathbb{R}$ are given by the equations

$$
h_{i}^{r}(z)=\frac{u_{i}\left(E\left(x_{\bullet}^{r}\right)+\left(1-\delta^{r}\right) z\right)-\delta^{r} y^{r}}{1-\delta^{r}} \text { and } h_{i}(z)=\left\langle d u_{i}(x), z\right\rangle+u_{i}(x) .
$$


The function $h_{i}$ is obviously concave. Furthermore, take a point $\bar{x}$ such that $u_{i}(\bar{x})>0$ for each $i \in N$ (the existence of such a point is guaranteed by Assumption (A2)). Then by the concavity of the utility functions we have $0<u_{i}(\bar{x}) \leq u_{i}(x)+\left\langle d u_{i}(x), \bar{x}-x\right\rangle=h_{i}(\bar{x}-x)$ for each $i$. It remains to verify that $h_{i}^{r}\left(z^{r}\right) \longrightarrow h_{i}(z)$ whenever $z^{r} \longrightarrow z$.

Write

$$
\begin{gathered}
x^{r}=E\left(x_{\bullet}^{r}\right)+\left(1-\delta^{r}\right) z^{r} \text { and } \\
h_{i}^{r}\left(z^{r}\right)=\frac{u_{i}\left(x^{r}\right)-\delta^{r} y_{i}^{r}}{1-\delta^{r}}=\frac{u_{i}\left(x^{r}\right)-y_{i}^{r}}{1-\delta^{r}}+y_{i}^{r} .
\end{gathered}
$$

Since $y_{i}^{r} \longrightarrow u_{i}(x)$ we only have to show that $\left[u_{i}\left(x^{r}\right)-y_{i}^{r}\right] /\left(1-\delta^{r}\right) \longrightarrow\left\langle d u_{i}(x), z\right\rangle$. To do so we give an upper and a lower bound on $\left[u_{i}\left(x^{r}\right)-y_{i}^{r}\right] /\left(1-\delta^{r}\right)$ and show that both converge to $\left\langle d u_{i}(x), z\right\rangle$.

For each $k \in N$ we have the inequalities

$$
u_{i}\left(x^{r}\right)-u_{i}\left(x_{k}^{r}\right) \geq\left\langle d u_{i}\left(x^{r}\right), x^{r}-x_{k}^{r}\right\rangle .
$$

Taking the expected value of both sides of the inequality with respect to $k$ and dividing by $1-\delta^{r}$ yields

$$
\frac{u_{i}\left(x^{r}\right)-y_{i}^{r}}{1-\delta^{r}} \geq \frac{\left\langle d u_{i}\left(x^{r}\right), x^{r}-E\left(x_{\bullet}^{r}\right)\right\rangle}{1-\delta^{r}}=\left\langle d u_{i}\left(x^{r}\right), z^{r}\right\rangle \longrightarrow\left\langle d u_{i}(x), z\right\rangle .
$$

Let $d_{i j}^{r}$ be as in Proposition 4.1. Since the sequence is bounded for each $(i, j) \in S \times S$ we can assume without loss of generality that it converges to the point $d_{i j}$. Notice that

$$
\sum_{i \in S} \sum_{j \in S} \mu_{i} \mu_{j} d_{i j}^{r}=0, \text { therefore } \sum_{i \in S} \sum_{j \in S} \mu_{i} \mu_{j} d_{i j}=0 .
$$

Now for each $k \in N$ we have the inequality

$$
\begin{aligned}
u_{i}\left(x^{r}\right)-u_{i}\left(x_{k}^{r}\right) & \leq\left\langle d u_{i}\left(x_{k}^{r}\right), x^{r}-x_{k}^{r}\right\rangle \\
& =\left\langle d u_{i}\left(x_{k}^{r}\right), x^{r}-E\left(x_{\bullet}^{r}\right)\right\rangle-\left\langle d u_{i}\left(x_{k}^{r}\right), x_{k}^{r}-E\left(x_{\bullet}\right)\right\rangle \\
& =\left\langle d u_{i}\left(x_{k}^{r}\right), x^{r}-E\left(x_{\bullet}^{r}\right)\right\rangle-\sum_{j \in S} \mu_{j}\left\langle d u_{i}\left(x_{k}^{r}\right), x_{k}^{r}-x_{j}^{r}\right\rangle .
\end{aligned}
$$

Taking the expected value with respect to $k$ we get

$$
u_{i}\left(x^{r}\right)-y_{i} \leq \sum_{k \in S} \mu_{k}\left\langle d u_{i}\left(x_{k}^{r}\right), x^{r}-E\left(x_{\bullet}^{r}\right)\right\rangle-\sum_{k \in S} \sum_{j \in S} \mu_{k} \mu_{j}\left\langle d u_{i}\left(x_{k}^{r}\right), x_{k}^{r}-x_{j}^{r}\right\rangle .
$$

Dividing by $1-\delta^{r}$ and taking the limit we get

$$
\begin{aligned}
\frac{u_{i}\left(x^{r}\right)-y_{i}}{1-\delta^{r}} & \leq \sum_{k \in N} \mu_{k}\left\langle d u_{i}\left(x_{k}^{r}\right), z^{r}\right\rangle-\sum_{k \in S} \sum_{j \in S} \mu_{k} \mu_{j}\left\langle d u_{i}\left(x_{k}^{r}\right), d_{k j}^{r}\right\rangle \\
& \longrightarrow\left\langle d u_{i}(x), z\right\rangle-\sum_{k \in S} \sum_{j \in S} \mu_{k} \mu_{j}\left\langle d u_{i}(x), d_{k j}\right\rangle \\
& =\left\langle d u_{i}(x), z\right\rangle-\left\langle d u_{i}(x), \sum_{k \in S} \sum_{j \in S} \mu_{k} \mu_{j} d_{k j}\right\rangle \\
& =\left\langle d u_{i}(x), z\right\rangle .
\end{aligned}
$$


This completes the proof.

Define the correspondences $\varphi_{i}: \mathbb{R}^{m} \rightrightarrows \mathbb{R}^{m}$ by the equations

$$
\varphi_{i}(x)=\arg \max _{z \in Z(x)}\left\langle d u_{i}(x), z\right\rangle \text { for each } i \in S .
$$

When the set $Z(x)$ is not bounded, the function $z \mapsto\left\langle d u_{i}(x), z\right\rangle$ may attain no maximum on $Z(x)$, in which case the set $\varphi_{i}(x)$ is empty. For future reference we state the following observation.

Proposition 4.4 Let $z \in \varphi_{i}(x)$. Then $\left\langle d u_{i}(x), z\right\rangle+u_{i}(x)>0$.

Proof. Take a point $\bar{x}$ such that $u_{k}(\bar{x})>0$ for each $k \in N$ (the existence of such a point is guaranteed by Assumption (A2)). Then by the concavity of the utility functions we have $0<u_{k}(\bar{x}) \leq u_{k}(x)+\left\langle d u_{k}(x), \bar{x}-x\right\rangle$ for each $k$. Then the point $\bar{x}-x$ is an element of $Z(x)$ and the result follows.

Proposition 4.5 For each $r$ let $\left(x_{\bullet}^{r}, A_{\bullet}^{r}\right)$ be a bargaining equilibrium of the game $\Gamma\left(\delta^{r}\right)$ inducing the continuation payoffs $y_{\bullet}^{r}$ and the social acceptance set $A^{r}$. Suppose that $\delta^{r} \longrightarrow 1$ and $x^{r} \longrightarrow x$ for $x^{r} \in A^{r}$. Let

$$
z_{i}^{r}=\left[x_{i}^{r}-E\left(x_{\bullet}^{r}\right)\right] /\left(1-\delta^{r}\right)
$$

If $z_{i}^{r} \longrightarrow z_{i}$ then $z_{i} \in \varphi_{i}(x)$.

Proof. Let the set $Z^{r}$ be as in Proposition 4.3. It is clear that $z_{i}^{r} \in Z^{r}$ for each $n$ and consequently $z_{i} \in Z(x)$. Now suppose that $z_{i}$ is not an element of $\varphi_{i}(x)$. Then there exists a point $z^{\prime}$ in $Z(x)$ such that $\left\langle d u_{i}(x), z_{i}\right\rangle<\left\langle d u_{i}(x), z^{\prime}\right\rangle$. By Proposition 4.3 there exists a sequence of points $z^{r}$ converging to $z$ such that $z^{r} \in Z^{r}$ for each $n$. Let $x^{r}$ be a point of $A^{r}$ such that $z^{r}=\left[x^{r}-E\left(x_{\bullet}\right)\right] /\left(1-\delta^{r}\right)$. For $n$ large enough we have the inequality $\left\langle d u_{i}\left(x^{r}\right), z_{i}^{r}\right\rangle<$ $\left\langle d u_{i}\left(x^{r}\right), z^{r}\right\rangle$. Rearranging this we obtain $\left\langle d u_{i}\left(x^{r}\right), x_{i}^{r}\right\rangle<\left\langle d u_{i}\left(x^{r}\right), x^{r}\right\rangle$. Finally, the concavity of the function $u_{i}$ gives the inequality $u_{i}\left(x_{i}^{r}\right)-u_{i}\left(x^{r}\right) \leq\left\langle d u_{i}\left(x^{r}\right), x_{i}^{r}-x^{r}\right\rangle<0$, which contradicts the fact that $x_{i}^{r}$ maximizes the function $u_{i}$ on the set $A^{r}$.

Define the correspondence $\varphi: \mathbb{R}^{m} \rightrightarrows \mathbb{R}^{m}$ by the equation

$$
\varphi(x)= \begin{cases}\sum_{i \in S} \mu_{i} \varphi_{i}(x) & \text { if } \varphi_{i}(x) \neq \oslash \text { for each } i \in S \\ \oslash & \text { otherwise. }\end{cases}
$$

We shall refer to $\varphi$ as the linearization correspondence. We are now in a position to state the main result of the section.

Theorem 2 If $x$ is a limit of a sequence of bargaining equilibria then $0 \in \varphi(x)$. 
Proof. There exists a sequence of bargaining equilibria $\left(x_{\bullet}^{r}, A_{\bullet}^{r}\right)$ of the game $\Gamma\left(\delta^{r}\right)$ inducing the social acceptance set $A^{r}$ such that $x^{r} \longrightarrow x$ for each sequence of points $x^{r} \in A^{r}$. Let $d_{i j}^{r}$ be as in Proposition 4.1 and $z_{i}^{r}$ be as in Proposition 4.5. We know that for each pair $(i, j) \in S \times S$ the sequence $\left\{d_{i j}^{r}\right\}$ is bounded. It follows that for each $i \in S$ the sequence $\left\{z_{i}^{r}\right\}$ is bounded, for we can write $z_{i}^{r}=\sum_{j \in S} \mu_{j} d_{i j}^{r}$. Therefore, replacing the sequences by subsequences, we can assume that $z_{i}^{r} \longrightarrow z_{i}$ for each $i \in S$. Since $\sum_{i \in S} \mu_{i} z_{i}^{r}=0$ each $n$, in the limit $\sum_{i \in S} \mu_{i} z_{i}=0$. And since $z_{i}$ is an element of $\varphi_{i}(x)$ by Proposition 4.5, zero is an element of $\varphi(x)$.

\section{The one-dimensional space of alternatives}

In this section we establish the asymptotic uniqueness of bargaining equilibria in the case $m=1$. This result is subsumed by the asymptotic uniqueness result in a companion paper by Predtetchinski [14] which only deals with the one-dimensional model. In the companion paper the acceptance or a rejection of a proposal is determined by a general acceptance rule represented by the collection of decisive coalitions. Cardona and Ponsatí [4] establish the asymptotic uniqueness of bargaining equilibria in the one-dimensional model where the proposer is chosen according to some deterministic rule, while Herings and Predtetchinski [8] consider a game where the identity of the proposer follows a Markov process.

We continue to maintain Assumptions (A1) through (A4). In particular Assumption (A4) requires that there be no point at which the derivatives of all utility functions vanish. We supplement our Assumptions (A1)-(A4) by the additional condition which requires that the derivative of each utility function vanishes at one point at most. In other words each player must have only one bliss point or none at all. We shall write $u_{i}^{\prime}(x)$ rather than $d u_{i}(x)$ to denote the derivative of the function $u_{i}$ at $x$.

(A5) For each $i$ there is at most one point $x$ in $\mathbb{R}$ such that $u_{i}^{\prime}(x)=0$.

Theorem 3 Suppose $m=1$. Assume (A1)-(A5). Then the correspondence $\varphi$ has a unique zero. Consequently, bargaining equilibria are asymptotically unique.

We prove Theorem 3 by showing that the linearization correspondence $\varphi$ is a decreasing correspondence. We say that a correspondence $\gamma: \mathbb{R} \rightrightarrows \mathbb{R}$ is decreasing if $y>\bar{y}$ whenever $y \in \gamma(x), \bar{y} \in \gamma(\bar{x})$ and $x<\bar{x}$. It is immediate that a decreasing correspondence has at most one zero point.

Given a point $x \in \mathbb{R}^{m}$ define the (possibly empty) sets

$$
\begin{aligned}
N_{-}(x) & =\left\{i \in N \mid u_{i}^{\prime}(x)<0\right\} \\
N_{o}(x) & =\left\{i \in N \mid u_{i}^{\prime}(x)=0\right\} \\
N_{+}(x) & =\left\{i \in N \mid u_{i}^{\prime}(x)>0\right\} .
\end{aligned}
$$

Assumption (A4) tells us that there is no point $x$ where the derivatives of all the functions $u_{i}$ vanish. Therefore, for each $x$ either the set $N_{-}(x)$ or the set $N_{+}(x)$ is non-empty. The 
set $Z(x)$ is given by

$$
\begin{gathered}
Z(x)= \begin{cases}{\left[\varphi_{-}(x),+\infty\right)} & \text { if } N_{-}(x)=\oslash \text { and } N_{+}(x) \neq \oslash \\
{\left[\varphi_{-}(x), \varphi_{+}(x)\right]} & \text { if } N_{-}(x) \neq \oslash \text { and } N_{+}(x) \neq \oslash \text { where } \\
\left(-\infty, \varphi_{+}(x)\right] & \text { if } N_{-}(x) \neq \oslash \text { and } N_{+}(x)=\oslash,\end{cases} \\
\varphi_{-}(x)=-\min _{i \in N_{+}(x)}\left\{u_{i}(x) / u_{i}^{\prime}(x)\right\} \text { and } \varphi_{+}(x)=-\max _{i \in N_{-}(x)}\left\{u_{i}(x) / u_{i}^{\prime}(x)\right\} .
\end{gathered}
$$

For each player $i$ in the set $N_{-}(x)$ the maximization of the function $z \mapsto u_{i}^{\prime}(x) z$ on the set $Z(x)$ is equivalent to the minimization of $z$ and yields the lower endpoint of $Z(x)$. For a player $i$ in $N_{+}(x)$ the maximization of $z \mapsto u_{i}^{\prime}(x) z$ is equivalent to the maximization of $z$ and yields the upper endpoint of $Z(x)$. Thus

$$
\varphi_{i}(x)= \begin{cases}\oslash & \text { if } i \in N_{+}(x) \text { and } N_{-}(x)=\oslash \\ \left\{\varphi_{+}(x)\right\} & \text { if } i \in N_{+}(x) \text { and } N_{-}(x) \neq \oslash \\ Z(x) & \text { if } i \in N_{o}(x) \\ \left\{\varphi_{-}(x)\right\} & \text { if } i \in N_{-}(x) \text { and } N_{+}(x) \neq \oslash \\ \oslash & \text { if } i \in N_{-}(x) \text { and } N_{+}(x)=\oslash .\end{cases}
$$

Proposition 5.1 The correspondences $\varphi_{i}$ for each $i \in N$ and the correspondence $\varphi$ are decreasing correspondences.

Proof. First we show that the function $\varphi_{-}$is a decreasing function. Let $x<\bar{x}$ and suppose that $N_{+}(x) \neq \oslash$ and $N_{+}(\bar{x}) \neq \oslash$. Then we have the inequalities

$$
\begin{aligned}
-\varphi_{-}(x) & =\min _{i \in N_{+}(x)}\left\{u_{i}(x) / u_{i}^{\prime}(x)\right\} \\
& \leq \min _{i \in N_{+}(\bar{x})}\left\{u_{i}(x) / u_{i}^{\prime}(x)\right\} \\
& <\min _{i \in N_{+}(\bar{x})}\left\{u_{i}(\bar{x}) / u_{i}^{\prime}(\bar{x})\right\}=-\varphi_{-}(\bar{x}) .
\end{aligned}
$$

The first inequality is true because $N_{+}(x) \supset N_{+}(\bar{x})$. To see that the second inequality is true take an $i \in N_{+}(\bar{x})$. Then $u_{i}^{\prime}(\bar{x})>0$. Therefore, $u_{i}(x)<u_{i}(\bar{x})$ and $u_{i}^{\prime}(x) \geq u_{i}^{\prime}(\bar{x})$. It follows that $u_{i}(x) / u_{i}^{\prime}(x)<u_{i}(\bar{x}) / u_{i}^{\prime}(\bar{x})$.

We conclude that $\varphi_{-}(x)>\varphi_{-}(\bar{x})$. Similarly, one proves that $\varphi_{+}$is a decreasing function.

Now we show that each $\varphi_{i}$ is a decreasing correspondence. Let $x<\bar{x}, z \in \varphi_{i}(x)$ and $\bar{z} \in \varphi_{i}(\bar{x})$. Since $u_{i}$ is a concave function, $u_{i}^{\prime}(x) \geq u_{i}^{\prime}(\bar{x})$. Since by Assumption (A5) the derivative of each function $u_{i}$ becomes zero at one point at most, either (a) $u_{i}^{\prime}(x)>0$ or (b) $u_{i}^{\prime}(\bar{x})<0$. In case (a) we have $z=\varphi_{+}(x)>\varphi_{+}(\bar{x}) \geq \bar{z}$. And in case (b) we have $z \geq \varphi_{-}(x)>\varphi_{-}(\bar{x})=\bar{z}$. 


\section{The asymmetric Nash bargaining solution}

In this section we show that if $m=n-1$ then every sequence of bargaining equilibria converges to the asymmetric Nash bargaining solution. This result is equivalent to that in Laruelle and Valenciano [3] and in Miyakawa [13]. In [3] and [13] the players propose the utility payoffs directly rather than the alternatives as in the game $\Gamma(\delta)$, but this difference is inessential. Indeed, one can see that if $m=n-1$ then the set of efficient utility payoffs in the game $\Gamma(\delta)$ satisfies all assumptions of Miyakawa [13].

Definition 3 The asymmetric Nash product is a function $\rho: X \rightarrow \mathbb{R}$ defined by $\rho(x)=$ $\times_{i \in S}\left(u_{i}(x)\right)^{\mu_{i}}$. The point $x$ of $X$ is the asymmetric Nash bargaining solution if it maximizes the function $\rho$ on the set $X$.

Theorem 4 Suppose $m=n-1$. Assume (A1)-(A4). Suppose moreover that $\mu_{i}>0$ for each $i \in N$. Then the asymmetric Nash bargaining solution is the unique zero of the correspondence $\varphi$. Consequently, the limit of every sequence of bargaining equilibria is the asymmetric Nash bargaining solution.

To derive the first order conditions for the maximization of the asymmetric Nash product $\rho$, observe that one can maximize instead the logarithm of the function $\rho$ on the set of alternatives $x$ such that $u_{i}(x)>0$ for each $i \in N$, this set being non-empty by (A2). The point $x$ is the asymmetric Nash bargaining solution if and only if

$$
u_{i}(x)>0 \text { for each } i \in N \text { and } \sum_{i \in N} \mu_{i} d u_{i}(x) / u_{i}(x)=0 .
$$

Let $d u(x)$ denote the $(n \times m)$-matrix with row $i$ given by $d u_{i}(x)$. Let $T(x)$ be the image of $d u(x)$. That is $T(x)$ is a linear subspace of $\mathbb{R}^{n}$ consisting of the points of the form $d u(x) z$ for $z \in \mathbb{R}^{m}$. By Assumption (A4) the matrix $d u(x)$ has rank $m$ and consequently $T(x)$ is of dimension $m$. Since $m=n-1$, the orthogonal complement $T^{\perp}(x)$ to $T(x)$ is a one-dimensional space. For each $x$ fix some non-zero vector $\ell(x)$ in $T^{\perp}(x)$. We shall take $\ell(x)$ to be a non-negative vector whenever such a vector is an element of $T^{\perp}(x)$. Thus we can write

$$
T(x)=\left\{t \in \mathbb{R}^{n} \mid\langle\ell(x), t\rangle=0\right\} .
$$

The first order conditions for the asymmetric Nash bargaining solution can now be restated in terms of the vector $\ell(x)$ as follows. Notice that by Equation (1) the vector $\left(\mu_{i} / u_{i}(x)\right)_{i \in N}$ is in the orthogonal complement to the space $T(x)$, and hence proportional to $\ell(x)$. Therefore, the point $x$ is the asymmetric Nash bargaining solution if and only if

$$
\ell_{i}(x)>0 \text { and } \ell_{i}(x) u_{i}(x)=b \mu_{i} \text { for all } i \in N \text { for some } b>0 .
$$

Proposition 6.1 describes the correspondence $\varphi$.

Proposition 6.1 Let $x \in \mathbb{R}^{m}$ and $z \in \varphi(x)$. Then $\ell_{j}(x)>0$ and $\left\langle d u_{j}(x), z\right\rangle+u_{j}(x)=$ $\mu_{j} b(x) / \ell_{j}(x)$ for each $j \in N$, where $b(x)>0$ is a real number. 
Proof. Let

$$
b(x)=\sum_{k \in N} \ell_{k}(x) u_{k}(x)
$$

Now take $z_{i} \in \varphi_{i}(x)$ and let $t_{i}=d u(x) z_{i}$ for each $i \in N$. We shall write $t_{i j}$ to denote the component $j$ of the vector $t_{i}$. It is obvious from the definition of the correspondence $\varphi_{i}$ and the definition of the space $T(x)$ that the point $t_{i}$ is a solution to the optimization problem

$$
\text { maximize } \bar{t}_{i i} \text { subject to }\left\langle\ell(x), \bar{t}_{i}\right\rangle=0 \text { and } \bar{t}_{i j}+u_{j}(x) \geq 0 \text { for each } j \in N \text {. }
$$

We must have $\ell_{i}(x)>0$ for otherwise the problem of player $i$ would have no solution. We have

$$
\begin{gathered}
t_{i j}=-u_{j}(x) \text { for each }(i, j) \in N \times N, i \neq j \\
t_{i i}=b(x) / \ell_{i}(x)-u_{i}(x) \text { for each } i \in N .
\end{gathered}
$$

By Proposition 4.4 we have $\left\langle d u_{i}(x), z_{i}\right\rangle>-u_{i}(x)$. Because $t_{i i}=\left\langle d u_{i}(x), z_{i}\right\rangle$ it follows immediately from the above equation that $b(x)>0$.

A typical element of $\varphi(x)$ is $z=\sum_{i \in N} \mu_{i} z_{i}$ with $z_{i}$ an element of $\varphi_{i}(x)$. We have

$$
\begin{aligned}
\left\langle d u_{j}(x), z\right\rangle & =\sum_{i \in N} \mu_{i}\left\langle d u_{j}(x), z_{i}\right\rangle \\
& =\sum_{i \in N} \mu_{i} t_{i j} \\
& =\mu_{j} b(x) / \ell_{j}(x)-\mu_{j} u_{j}(x)-\left(1-\mu_{j}\right) u_{j}(x) \\
& =\mu_{j} b(x) / \ell_{j}(x)-u_{j}(x) .
\end{aligned}
$$

The result follows.

The proof of Theorem 4 is now immediate. Suppose that $0 \in \varphi(x)$. Letting $z=0$ in Proposition 6.1 shows that the condition 2 is satisfied.

\section{Quadratic utilities}

In this section we show the asymptotic uniqueness of bargaining equilibria when the utility of each player is quadratic. To the best of my knowledge, it is the only result on the asymptotic uniqueness of bargaining equilibria outside the extreme cases $m=1$ and $m=$ $n-1$. More precisely, we impose the following assumptions.

(Q1) The set $N$ is a subset of $\mathbb{R}^{m}$ containing zero. Player 0 has positive recognition probability. Furthermore, if $0 \leq\langle i, z\rangle$ for all $i \in N$, then $z=0$.

(Q2) The utility function of player $i$ is given by

$$
u_{i}(x)=1-\langle x, x\rangle / 2+\langle i, x\rangle=1+\left(\|i\|^{2}-\|i-x\|^{2}\right) / 2 .
$$


Thus the point $i$ is the ideal point of player $i$ and the upper counter sets of the function $u_{i}$ are the balls centered at $i$. The gradient of the function $u_{i}$ is given by $d u_{i}(x)=i-x$. Assumption (Q1) guarantees that $z=0$ whenever $\left\langle d u_{i}(x), z\right\rangle=0$ for each $i \in N$, as is required by Assumption (A4).

Theorem 5 Assume (Q1) and (Q2). Then the correspondence $\varphi$ has a unique zero. Consequently, bargaining equilibria are asymptotically unique.

We need the following definitions.

$$
\begin{gathered}
S(x)=\bigcap_{i \in N}\left\{s \in \mathbb{R}^{m} \mid\langle x-i, s\rangle \leq 1\right\}, \\
a(x)=1+\langle x, x\rangle / 2 \text { and } j(x)=x / a(x) . \\
B=\left\{x \in \mathbb{R}^{m} \mid\langle x, x\rangle \leq 2\right\} .
\end{gathered}
$$

Also define the correspondence $\phi: \mathbb{R}^{m} \rightrightarrows \mathbb{R}^{m}$ by the following equations.

$$
\begin{gathered}
\phi(x)=\arg \max _{s \in S(x)}\langle i-x, s\rangle \text { for each } i \in N \\
\phi(x)= \begin{cases}\sum_{i \in S} \mu_{i} \phi_{i}(x) & \text { if } \phi_{i}(x) \neq \oslash \text { for each } i \in S \\
\oslash & \text { otherwise. }\end{cases}
\end{gathered}
$$

The following proposition is implied directly by our definitions.

Proposition 7.1 Assume (Q1) and (Q2). Suppose $0 \in \varphi(x)$. Then $(a) x \in B$ and $(b)$ $j(x) \in \phi(x)$.

Proof. If $0 \in \varphi(x)$, then $0 \in Z(x)$ and therefore $u_{i}(x) \geq 0$ for every $i \in N$. Since $0 \in N$ by assumption (Q1), we have $u_{0}(x)=1-\langle x, x\rangle / 2 \geq 0$, which proves part (a). To prove part (b), we compute the set $Z(x)$ :

$$
\begin{aligned}
Z(x) & =\cap\left\{z \in \mathbb{R}^{m} \mid\left\langle d u_{i}(x), z\right\rangle+u_{i}(x) \geq 0\right\} \\
& =\cap\left\{z \in \mathbb{R}^{m} \mid\langle i-x, z\rangle+1-\langle x, x\rangle / 2+\langle i, x\rangle \geq 0\right\} \\
& =\cap\left\{z \in \mathbb{R}^{m} \mid\langle i-x, z+x\rangle+1+\langle x, x\rangle / 2 \geq 0\right\} \\
& =\cap\left\{z \in \mathbb{R}^{m} \mid\langle x-i, z+x\rangle \leq a(x)\right\} \\
& =\cap\left\{z \in \mathbb{R}^{m} \mid z=a(x) s-x \text { for some } s \in S(x)\right\} \\
& =a(x) S(x)-\{x\} .
\end{aligned}
$$

It follows that $\varphi(x)=a(x) \phi(x)-\{x\}$. If $0 \in \varphi(x)$, then $x \in a(x) \phi(x)$, or equivalently $j(x)=x / a(x) \in \phi(x)$.

We say that the correspondence $\gamma: \mathbb{R}^{m} \rightrightarrows \mathbb{R}^{m}$ is increasing on the set $B$ if $\left.\langle x-\bar{x}, s-\bar{s}\rangle\right\rangle$ 0 for every $s \in \gamma(x)$ and $\bar{s} \in \gamma(\bar{x})$ and every pair of points $x \neq \bar{x}$ in $B$. It is weakly increasing if the inequality is weak. The correspondence $\gamma$ is (weakly) decreasing if $-\gamma$ is (weakly) increasing. We prove Theorem 5 by showing that the correspondence $\phi$ is weakly decreasing, while the function $j$ is increasing on the set $B$.

First we establish the monotonicity of the function $j$. 
Proposition 7.2 The function $j$ is increasing on the set $B$.

Proof. Take two distinct points $x$ and $\bar{x}$ in $B$. Suppose first that $\|x\| \neq\|\bar{x}\|$. Then

$$
\begin{aligned}
a(x) a(\bar{x})\langle x-\bar{x}, j(x)-j(\bar{x})\rangle & =\langle x-\bar{x}, a(\bar{x}) x-a(x) \bar{x}\rangle \\
& =a(\bar{x})\langle x, x\rangle+a(x)\langle\bar{x}, \bar{x}\rangle-(a(x)+a(\bar{x}))\langle x, \bar{x}\rangle \\
& \geq a(\bar{x})\|x\|^{2}+a(x)\|\bar{x}\|^{2}-(a(x)+a(\bar{x}))\|x\|\|\bar{x}\| \\
& =a(\bar{x})\|x\|(\|x\|-\|\bar{x}\|)+a(x)\|\bar{x}\|(\|\bar{x}\|-\|x\|) \\
& =(\|x\|-\|\bar{x}\|)(a(\bar{x})\|x\|-a(x)\|\bar{x}\|) \\
& =(\|x\|-\|\bar{x}\|)\left(\|x\|+\|x\|\|\bar{x}\|^{2} / 2-\|\bar{x}\|-\|\bar{x}\|\|x\|^{2} / 2\right) \\
& =(\|x\|-\|\bar{x}\|)(\|x\|-\|\bar{x}\|+\|x\|\|\bar{x}\|(\|\bar{x}\|-\|x\|) / 2) \\
& =(\|x\|-\|\bar{x}\|)^{2}(1-\|x\|\|\bar{x}\| / 2)>0 .
\end{aligned}
$$

The first inequality is implied by the Schwarz-Cauchy inequality $|\langle x, \bar{x}\rangle| \leq\|x\|\|\bar{x}\|$. The second inequality holds because $\|x\| \neq\|\bar{x}\|$ and $\|x\| \leq \sqrt{2}$ and $\|\bar{x}\| \leq \sqrt{2}$, of which at least one inequality is strict. Suppose now that $\|x\|=\|\bar{x}\|$. Then

$$
a(x) a(\bar{x})\langle x-\bar{x}, j(x)-j(\bar{x})\rangle=a(x)\langle x-\bar{x}, x-\bar{x}\rangle>0 .
$$

We now turn to the properties of the set $S(x)$ and the correspondence $\phi$. Given two points $x$ and $\bar{x}$ of $\mathbb{R}^{m}$ define the function $h=h[x, \bar{x}]: S(x) \longrightarrow \mathbb{R}^{m}$ by the equation

$$
h[x, \bar{x}](s)=\frac{s}{1-\langle x-\bar{x}, s\rangle} .
$$

Proposition 7.3 Let $x$ and $\bar{x}$ be two points of $\mathbb{R}^{m}$ such that $\phi(x) \neq \oslash$ and $\phi(\bar{x}) \neq \varnothing$. Then

(a) $\langle x-\bar{x}, s\rangle<1$ for all $s \in S(x)$ and $\langle\bar{x}-x, \bar{s}\rangle<1$ for all $\bar{s} \in S(\bar{x})$.

(b) The map $h[x, \bar{x}]$ carries the set $S(x)$ homeomorphically onto $S(\bar{x})$ and $h[\bar{x}, x]$ is its inverse.

Proof. (a) To prove the first inequality suppose there exists a point $s$ in $S(x)$ such that $1 \leq\langle x-\bar{x}, s\rangle$. We have $\langle x-i, s\rangle \leq 1$ for each $i \in N$. Suppose first that $0 \leq\langle\bar{x}, s\rangle$. Then $0 \leq\langle\bar{x}, s\rangle \leq\langle x, s\rangle-1 \leq\langle i, s\rangle$ for each $i \in N$ and Assumption (Q1) implies that $s=0$, a contradiction. We conclude that $\langle\bar{x}, s\rangle<0$. But now $\langle\bar{x}-i, s\rangle=\langle\bar{x}-x, s\rangle+\langle x-i, s\rangle \leq$ $-1+1=0$. Therefore, the point $t s$ is an element of the set $S(\bar{x})$ for each $t \geq 0$. But then $\phi_{0}(\bar{x})=\oslash$, because $-t\langle\bar{x}, s\rangle$ converges to plus infinity as $t$ goes to infinity. Since player 0 has positive recognition probability by Assumption (Q1), it follows that $\phi(\bar{x})=\oslash$ contrary to the assumption of the proposition. The second inequality follows by interchanging the roles of $x$ and $\bar{x}$. 
(b) We only have to show that $h[x, \bar{x}]$ carries the set $S(x)$ into the set $S(\bar{x})$ and that $h[\bar{x}, x] \circ h[x, \bar{x}]$ is the identity map on $S(x)$. The result then follows since $x$ and $\bar{x}$ are arbitrary. For simplicity we write $h$ for $h[x, \bar{x}]$ and $\bar{h}$ for $h[\bar{x}, x]$. For $s \in S(x)$ we have

$$
\begin{aligned}
\langle\bar{x}-i, h(s)\rangle & =\frac{\langle\bar{x}-i, s\rangle}{1-\langle x-\bar{x}, s\rangle} \\
& =\frac{\langle x-i, s\rangle-\langle x-\bar{x}, s\rangle}{1-\langle x-\bar{x}, s\rangle} \leq \\
& \leq \frac{1-\langle x-\bar{x}, s\rangle}{1-\langle x-\bar{x}, s\rangle}=1 .
\end{aligned}
$$

Thus the point $h(s)$ is an element of $S(\bar{x})$. We conclude that the map $h$ carries the set $S(x)$ into the set $S(\bar{x})$. Letting $c=1-\langle x-\bar{x}, s\rangle$ we see that

$$
(\bar{h} \circ h)(s)=\bar{h}(s / c)=\frac{s / c}{1-\langle\bar{x}-x, s / c\rangle}=\frac{s}{c-\langle\bar{x}-x, s\rangle}=s .
$$

We are now in a position to establish the monotonicity of the correspondence $\phi$.

Proposition 7.4 The correspondence $\phi$ is weakly decreasing.

Proof. We show that each $\phi_{i}$ is a weakly decreasing correspondence on the set of points $x$ such that $\phi(x) \neq 0$. Let $x$ and $\bar{x}$ be two points such that both $\phi(x)$ and $\phi(\bar{x})$ are nonempty. As before, we write $h$ for $h[x, \bar{x}]$ and $h[\bar{x}, x]$ for $\bar{h}$. Let $s \in \phi_{i}(x)$ and $\bar{s} \in \phi_{i}(\bar{x})$. Since the point $s$ is a maximizer of the function $\langle i-x, \bullet\rangle$ on the set $S(x)$ and since $\bar{h}(\bar{s})$ is one of its elements, we have the inequalities

$$
\langle i-x, \bar{h}(\bar{s})\rangle \leq\langle i-x, s\rangle .
$$

Since $\bar{h} \circ h$ is the identity map on the set $S(x)$, we can write $s=\bar{h}(h(s))$. Then the inequality above becomes

$$
\frac{\langle i-x, \bar{s}\rangle}{1-\langle\bar{x}-x, \bar{s}\rangle}=\langle i-x, \bar{h}(\bar{s})\rangle \leq\langle i-x, \bar{h}(h(s))\rangle=\frac{\langle i-x, h(s)\rangle}{1-\langle\bar{x}-x, h(s)\rangle} .
$$

Adding 1 to the left and the right hand side of this inequality then yields

$$
\frac{\langle i-\bar{x}, \bar{s}\rangle}{1-\langle\bar{x}-x, \bar{s}\rangle} \leq \frac{\langle i-\bar{x}, h(s)\rangle}{1-\langle\bar{x}-x, h(s)\rangle} .
$$

Since $\bar{s}$ is a maximizer of the function $\langle i-\bar{x}, \bullet\rangle$ on the set $S(\bar{x})$ and since $h(s)$ is one of its elements, we have the inequality

$$
\langle i-\bar{x}, \bar{s}\rangle \geq\langle i-\bar{x}, h(s)\rangle
$$


Notice that in (3) both denominators are positive by Proposition 7.3(a) and $\langle i-\bar{x}, \bar{s}\rangle>0$ because 0 is in the interior of $S(\bar{x})$. Therefore comparing Inequalities (3) and (4) gives

$$
1-\langle\bar{x}-x, \bar{s}\rangle \geq 1-\langle\bar{x}-x, h(s)\rangle .
$$

Rearranging we get

$$
\langle x-\bar{x}, h(s)-\bar{s}\rangle \leq 0
$$

Furthermore,

$$
\langle x-\bar{x}, s-h(s)\rangle=-\langle x-\bar{x}, s\rangle^{2} /(1-\langle x-\bar{x}, s\rangle) \leq 0 .
$$

Finally,

$$
\langle x-\bar{x}, s-\bar{s}\rangle=\langle x-\bar{x}, s-h(s)\rangle+\langle x-\bar{x}, h(s)-\bar{s}\rangle \leq 0 .
$$

This completes the proof.

\section{References}

[1] Jeffrey S. Banks And John Duggan: A Bargaining Model of Collective Choice. American Political Science Review, 94: 73-88, 2000.

[2] Ken Binmore, Ariel Rubinstein, and Asher Wolinsky: The Nash Bargaining Solution in Economic Modelling. The RAND Journal of Economics, 17: 176-188, 1986.

[3] Annick Laruelle and Federico Valenciano: Noncooperative Foundations of Bargaining Power in Committees and the Shapley-Shubik Index. Games and Economic Behavior, 63: 341-353, 2008.

[4] Daniel Cardona and Clara Ponsatí: Bargaining One-Dimensional Social Choices. Journal of Economic Theory, 137: 627-651, 2007.

[5] Seok-Ju Cho and John Duggan: Uniqueness of Stationary Equilibria in a OneDimensional Model of Bargaining. Journal of Economic Theory, 113: 118-130, 2003.

[6] Hülya ERaslan: Uniqueness of Stationary Equilibrium Payoffs in the BaronFerejohn Model. Journal of Economic Theory, 103: 11-30, 2002.

[7] Hülya Eraslan and Antonio Merlo: Majority Rule in a Stochastic Model of Bargaining. Journal of Economic Theory, 103: 31-48, 2002.

[8] P. Jean-Jacques Herings and Arkadi Predtetchinski: One-dimensional Bargaining with Markov Recognition Probabilities. Maastricht University, Research Memorandum RM/07/044. 
[9] Tasos Kalandrakis: Regularity of Pure Strategy Equilibrium Points in a Class of Bargaining Games. Economic Theory, 28: 309-329, 2006.

[10] Klaus Kultti and Hannu Vartiainen: Von Neumann-Morgenstern Stable Sets, Discounting, and Nash Bargaining. Journal of Economic Theory, 137: 721-728, 2007.

[11] Antonio Merlo and Charles Wilson: A Stochastic Model of Sequential Bargaining with Complete Information. Econometrica, 63: 371-399, 1995.

[12] Antonio Merlo and Charles Wilson: Effcient Delays in a Stochastic Model of Bargaining. Economic Theory, 11: 39-55, 1998.

[13] ToshiJi Miyakawa: Non-cooperative Foundation of $n$-Person Asymmetric Nash Bargaining Solution. Osaka University of Economics, working paper No. 2006-2.

[14] Arkadi Predtetchinski: One-Dimensional Bargaining With a General Voting Rule. Maastricht University, Research Memorandum RM/07/045. 